\section{AB0156 A NOVEL ASSOCIATION OF TLR-2 (23 BPINS/DEL: RS111200466) POLYMORPHISM WITH ANKYLOSING SPONDYLITIS - A POSSIBLE ROLE IN DISEASE SUSCEPTIBILITY: A HOSPITAL BASED CASE-CONTROL STUDY}

Abhisek Sabuii ${ }^{1}$, Rina Tripathy ${ }^{2}$, Aditya Kumar Panda ${ }^{3}$, Manoj Kumar Parida ${ }^{1}$, Saumya Ranjan Tripathy ${ }^{4}$, Bidyut Kumar Das ${ }^{1} .{ }^{1}$ S.C.B Medical College, Medicine, Cuttack, India; ${ }^{2}$ S.C.B Medical College, Biochemistry, Cuttack, India; ${ }^{3}$ Khallikote University, Life Science, Brahmapur, India; ${ }^{4}$ S.C.B Medical College, Rheumatology, Cuttack, India

Background: Role of innate immunity in pathogenesis of ankylosing spondylitis (AS) has been well documented ${ }^{1}$. Higher expression of TLR2 has been reported in AS patients and associated with clinical severity ${ }^{2}$. Recently, a functional $23 \mathrm{bp}$ ins/del polymorphism at 5'UTR of TLR2 gene has been reported and association of variant with elevated TLR2 surface expression and proinflammatory molecules has been elegantly demonstrated ${ }^{3}$. This is associated with high TNF-alpha levels, one of the key molecules in the pathogenesis of $\mathrm{AS}^{4}$. In this preliminary study, we investigated the possible role of TLR2 (23bp ins/del) polymorphism with AS in a cohort from Odisha, India.

Objectives: To investigate the role of TLR-2 (23 bp ins/del: rs111200466) Polymorphism in Ankylosing Spondylitis

Methods: AS patients $(n=101)$, who fulfilled the ASAS classification criteria for axial spondyloarthritis or ASAS classification criteria for peripheral spondyloarthritis were enrolled along with 100 healthy age matched controls from similar geographical areas. Patients were examined in detail and BASDAI/BASFI recorded. TLR2 (23 bp ins/del) polymorphism was genotyped by polymerase chain reaction. Genotype and allele distribution among patients and controls were compared by Fisher's exact test.

Results: All patients enrolled in the present study were males. The mean age of AS patients and healthy controls was $31.21 \pm 11.43$ and $28.28 \pm 9.62$ years, respectively. At the time of enrolment, mean disease duration of patients was $2.07 \pm 1.13$ years. BASDAI and BASFI scores were above 5. Distribution of TLR2 (23 bp ins/ del) polymorphism was in accordance with Hardy-Weinberg Equilibrium. Prevalence of del/del genotype was significantly higher in AS patients compared to healthy controls $(P=0.01, O R=5.65)$, indicating a possible contributory role of TLR2 on predisposition to AS. Distribution of heterozygous genotype (ins/del) and minor allele (del) were comparable among different clinical categories. Furthermore, no significant association of TLR-2 polymorphism was observed with disease severity.

Conclusion: TLR2 5'UTR homozygous mutants (23 bp deletion) were significantly associated with patients of AS in but not with disease severity. Larger sample size and levels of TNF alpha and IL17 in the mutants will further improve the understanding of its role in AS.

\section{REFERENCES}

[1] Vanaki N, Aslani S, Jamshidi A, Mahmoudi M. Role of innate immune system in the pathogenesis of ankylosing spondylitis. Biomedicine \& Pharmacotherapy. 2018 Sep 30;105:130-43.

[2] McCormack WJ, Parker AE, O'Neill LA. Toll-like receptors and NOD-like receptors in rheumatic diseases. Arthritis research \& therapy. 2009 Oct;11 (5):243.

[3] Das B, Tripathy R, Pattanaik S, Panda A. 270 Association of trr2 (23bp ins/del) polymorphism with systemic lupus erythematosus (sle) and p. falciparum malaria: a study in malaria endemic area of odisha, india.

[4] Blandizzi C, Gionchetti P, Armuzzi AL, Caporali R, Chimenti S, Cimaz R, Cimino L, Lapadula G, Lionetti P, Marchesoni A, Marcellusi A. The role of tumour necrosis factor in the pathogenesis of immune-mediated diseases. International journal of immunopathology and pharmacology. 2014 Jan;27 (1_suppl):1-0.

Disclosure of Interests: None declared

DOI: 10.1136/annrheumdis-2019-eular.5566

\section{AB0157 SYNOVIAL FLUID PROTEOMICS IN PATIENTS WITH ANKYLOSING SPONDYLITIS}

Chang-Nam Son ${ }^{1}$, Jihyun Lee ${ }^{1}$, Byeong-Wook Song ${ }^{1}$, Hye-Jin Jeong ${ }^{1}$, JinNyeong Chae', Ji-Min Kim', Sang-Hyon Kim ${ }^{1}$, Jinseol Rhee ${ }^{2}$, Jong-Seo Kim ${ }^{3}$. ${ }^{1}$ Keimyung University, Daegu, Korea, Rep. of (South Korea); ${ }^{2}$ Keimyung University Dongsan Medical Center, Daegu, Korea, Rep. of (South Korea); ${ }^{3}$ Center for RNA Research, Institute of Basic Science, Daegu, Korea, Rep. of (South Korea)

Background: Ankylosing Spondylitis (AS), affecting $0.5 \%$ of the population, is a chronic inflammatory rheumatic disease affecting the axial skeleton and peripheral joints. When peripheral arthritis in ankylosing spondylitis (AS) develops early in the disease course it is a predictor of more aggressive disease. SF is in contact with the primary tissues affected by arthritic diseases and has been implicated in disease pathophysiology. Therefore it is an excellent source for discovery of biomarkers.

Objectives: We used proteomic analysis using SFs from AS patients and other arthritis patients in order to discover novel diagnostic markers for AS. Our aim was to identify differentially expressed protein mediators in synovial fluid of ankylosing spondylitis.

Methods: A Total of $40 \mathrm{SF}$ samples from $10 \mathrm{AS}$ and each 10 controls [Osteoarthritis $(\mathrm{OA})$, Rheumatoid Arthritis (RA), gouty arthritis (Gout)] were collected. Liquid chromatography and tandem mass spectrometry (LC-MS/MS), to identify differentially expressed proteins based on the ratios of the extracted ion current of each protein between the four groups. Among the 9 proteins showing 1.5 fold change, 8 were verified with the exception of the abundant protein Haptoglobin (HP). Matrix metalloproteinase-1(MMP1) and Matrix metalloproteinase-3(MMP3) were used as a positive control, and the remaining 6 proteins were subjected to western blot analysis.

Results: We identified 9 proteins that were found to be more than 1.5 -fold differentially expressed in SF of AS patients compared to control groups. Proteins such as HP, MMP1, MMP3, Serum amyloid P-component(APCS), Complement facto H-related protein 5(CFHR5), Fumarylacetoacetase(FAH), Mannose-binding lectin2(MBL2), Complement component C9(C9) and Complement C4-A(C4A) were found to be upregulated in the SF of AS patients. CFHR5 and C9 were reported in previous studies with AS serum. APCS was reported in SF as well as serum. How ever, $\mathrm{FAH}, \mathrm{C} 4 \mathrm{~A}$ and MBL2 were newly discovered through this analysis. We were able to verify the unique expression level of C9 and CFHR5 in AS sample using western blot analysis compared to the other three diseases.

Conclusion: We performed quantitatively proteomic profiling of the respective SF sample from 4 diseases, i.e., AS, OA, RA, and GOUT, by LC-MS/MS. The systematic comparative proteomic analysis of the four groups together was carried out for the first time, leading to several differentially expressed proteins in AS Among them, we expect $\mathrm{C} 9$ and CFHR5, which expression levels were confirmed by western blot analysis, can be a potential biomarker for AS.

\section{REFERENCES}

[1] Fischer R, et al. Mol Cell Proteomics 2012;11:M111.013904.

[2] Bhattacharjee M, et al. Clinical Proteomics 2013;10:11

Acknowledgement: This research was supported by the National Research Foundation of Korea (NRF) funded by the Ministry of Science, ICT \& Future Planning (2017R1C1B5017278)

Disclosure of Interests: None declared

DOI: 10.1136/annrheumdis-2019-eular.2017

\section{AB0157B FREQUENCY AND SPECTRUM OF ANEMIC SYNDROME IN PATIENTS WITH ANKYLOSING SPONDYLITIS, PECULIARITIES OF CYTOMETRIC CHARACTERISTICS AND HEMOPOIESIS}

Sergii Shevchuk, Oxana Zviahina. National Pirogov Memorial Medical University, Vinnytsya, internal medicine, Vinnytsya, Ukraine

Background: Anemia is one of the most common extraarticular manifestations in patients with ankylosing spondylitis (AS). According to various sources, from 18.5 to $45.8 \%$ of patients with AS have anemia. In the pathogenesis of anemic syndrome in the AS, a leading role is given to proinflammatory cytokines (IL-1, IL-6, and TNF-alpha), which is associated with the development of anemia of chronic disease (ACD) in this category of patients. Another type of anemia in patients with AS is iron deficiency anemia (IDA), and the relationship between the latter and the $A C D$ varies significantly according to various literature data. As for other types of anemia in the AS, they are represented by anemia caused by drugs.

Objectives: The purpose of the work was to investigate the prevalence of anemia in the Ukrainian population of patients with AS and evaluate the hematopoiesis in patients with the main types of it.

Methods: The group with anemia included patients whose haemoglobin levels were below $120 \mathrm{~g} / \mathrm{l}$. The diagnosis of AS was determined according to the modified New York criteria (1984). Laboratory methods of research (general analysis of blood, erythrocytes, haemoglobin, color index, serum iron, total iron binding capacity (TIBC) included in the list of standard examinations of patients were performed according to standard methods. To verify the diagnosis of ACD, ferritin (FN) and levels of soluble transferrin receptor (sTfR) were determined.

Results: 118 patients with AS were included into the study, 11 (32.3\%) females and $23(67.7 \%)$ males. It was found that 34 patients $(28.8 \%)$ had anemia. Anemia of mild degree was manifested in $27(79.4 \%)$ patients and with moderate severity $7(20.6 \%)$ patients. Among 34 patients with anemia, patients with ACD - 15 $(44.1 \%)$ predominated. In the second place, $10(29.4 \%)$ showed a combination of ACD with functional deficiency of iron, and 8 (23.5\%) patients had signs of IDA. Only one of the subjects had signs of scarce anemia. Consequently, anemic 
syndrome in patients with AS is represented by ACD, ACD with iron deficiency and the actual IDA.

The analysis did not reveal significant differences in hemoglobin levels in the groups of patients with different types of anemia, depending on gender, age and duration of the disease.

The patients with different types of anemic syndrome did not have significant differences in haemoglobin content, the number of red blood cells and the magnitude of the $\mathrm{MCH}$, but significantly differed in magnitude MCV. The lowest values of MCV $(73.5 \pm 3.14 \mathrm{ft})$ were in patients with IDA, intermediate $(86.22 \pm 2.6 \mathrm{ft})$ in the group of patients with ACD with functional deficiency of iron and the highest $(94.2 \pm 1.68$ flask) among people with ACD $(p<0.05)$. According to cytometric distribution, $A C D$ was mostly (80\%) normocytic, $13.3 \%$ macrocytic and only $6.65 \%$ microcytic. ACD with functional deficiency of iron was normocytic in $70 \%$ of cases, in $20 \%$ and $10 \%$ of cases, microcytic and macrocytic, respectively. Microcytic changes of erythrocytes most often in $75 \%$ were registered in the group of patients with IDA.

The course of the AS was accompanied by significant violations of iron exchange. In particular, with anemia of the first degree, iron levels were $10.25 \pm 0.26 \mathrm{mmol} / \mathrm{l}$ and with anemia of the second degree $9.30 \pm 0.63 \mathrm{mmol} / \mathrm{l}(p<0.05)$. With regard to the types of anemic syndrome, in patients with IDA, serum iron levels were 8.30 $\pm 0.21 \mathrm{mmol} / \mathrm{l}$, or it was probably $(38 \%)$ lower than in patients with "pure" ACD. It was at the IDA that the total iron binding capacity (TIBC) was the highest. Patients with a combination of ACD with functional deficiency of iron with this indicator occupied an intermediate position between patients with IDA and ACD.

Conclusion: Thus, the most widespread variants of anemia in patients with AS are $A C D, I D A$ and $A C D$ with iron deficiency. ACD is in most cases a normocytic anemia, IDA - microcytic anemia, and ACD with iron deficiency mostly normocytic as well.

Disclosure of Interests: None declared

DOI: 10.1136/annrheumdis-2019-eular.4365

\section{AB0157C OCULAR CAUSES OF THE INITIATION OF SYSTEMIC THERAPYIN PATIENTS WITH SPONDYLOARTHRITIS (SPA) AND UVEITIS}

Marta Garijo Bufort ${ }^{1}$, Carlota Iñiguez ${ }^{2}$, Ana Garrote ${ }^{3}$, Carolina Álvarez Castro ${ }^{4}$, Miguel Cordero-Coma ${ }^{5}{ }^{1}$ Teaching Hospital of Valladolid, Rheumatology, Valladolid, Spain; ${ }^{2}$ University Health Care Complex of Leon, Rheumatology, León, Spain; ${ }^{3}$ University Health Care Complex of Leon., Ophthalmology, León, Spain; ${ }^{4}$ University Health Care Complex of Leon, Rheumatology, León, Spain; ${ }^{5}$ University Health Care Complex of Leon, Ophthalmology, Leon, Spain

Background: Uveitis is the most frequent extra-axial manifestation in Spondyloarthritis $(\mathrm{SpA})$. Even though, the criteria for the initiation of systemic therapy in patients with $\mathrm{SpA}$ are well defined, they are not when dealing with eye involvement. Objectives: The main objective of this study is to determine which patients require treatment with systemic therapy (ST), understood as such the diseasemodifying drugs (sulfasalazine and methotrexate) and the biological drugs (antiTNF- $\alpha$ ), due to ocular involvement.

Methods: This is an observational retrospective study carried out in the University Health Care Complex of León with patients diagnosed with SpA who have some episode of uveitis throughout the course of their disease and who are being treated with ST.

Results: We studied 28 patients, $67.9 \%$ men and $32.1 \%$ women, with a mean age at diagnosis of $34.48 \pm 12.69$ years. All patients had axial involvement and $64.3 \%$ had peripheral involvement. The initial ST was sulfasalazine (SSZ) in $50 \%$ of the patients, methotrexate (MTX) in $21.4 \%$ and anti-TNF $\alpha$ in $28.6 \%$. Within those treated with antiTNF $\alpha, 50 \%$ was treated with adalimumab and the other $50 \%$ with golimumab (25\%) and infliximab (25\%). A $78.57 \%$ of the patients required a second systemic therapy to control the disease; a $50 \%$ required a third treatment and only 3 patients needed a fourth systemic drug that, in all cases, was adalimumab. The patients who started the ST were exclusively due to uveitis in $32.1 \%$ and both due to uveitis and SpA in $35.7 \%$. When they need a second systemic treatment, it is still owing to an ophthalmological cause in $28.6 \%$ of the patients, and both due to ocular activity and SpA, in 33.4\%. Nevertheless, when these patients require a third anti-TNF $\alpha$, it is usually due to SpA (57.1\%). A $10.7 \%$ of patients requiring a fourth ST was because of uveitis. $66.7 \%$ of the patients who started the ST due to uveitis were treated that way because of the uveitis severity. $60.7 \%$ of the patients with uveitis presented complications in the form of vitritis $(70.6 \%)$, panuveitis $(11.8 \%)$, hypopyon $(5.9 \%)$, keratic precipitates $(35.3 \%)$, fibrin $(41.2 \%)$, synechiae $(5.9 \%)$, pupillary membrane $(23.5 \%)$, IOP increase $(41.2 \%)$ and cataract $(17.6 \%)$. The macular involvement was observed in $17.9 \%$ of the cases in the form of macular oedema $(40 \%)$, papillary oedema $(40 \%)$, epiretinal atrophy $(20 \%)$, neurosensory detachment $(20 \%)$ and epiretinal membrane $(40 \%)$. In $61.9 \%$ of the patients, the ST started due to refractoriness, with the average number of uveitis after the initiation of systemic therapy of $4.32 \pm 2.25$ uveitis. When exclusive eye involvement is present, the treatment with SSZ is usually initiated ( 6 patients), although 2 patients required from the start the anti-TNF $\alpha$ and 1 MTX. The average number of uveitis in patients with BASDAI $<4$ is $5.27 \mathrm{Cl} 95 \%$
(3.90-6.63) versus $3.23 \mathrm{Cl} 95 \%(2.41-4.05)$ of patients with BASDAl> $4(p<0.05)$; while in patients without peripheral involvement is of $5.60 \mathrm{Cl} 90 \%(3.75-6.85) \mathrm{com}$ pared to 3.78 of the patients with peripheral involvement $\mathrm{Cl} 90 \%(2.71-4.85)$ ( $p$ $<0.05)$.

Conclusion: The treatment of uveitis with ST in a patient with SpA is determined by the presence of complications and/or by the refractoriness of these, being the SSZ the most used drug at the beginning. It seems that the activity of the SpA is independent of uveitis and that patients with purely axial forms have more uveitis than those with peripheral involvement.

Disclosure of Interests: None declared

DOI: 10.1136/annrheumdis-2019-eular.6732

\section{SLE, Sjögren's and APS - etiology, pathogenesis and animal models}

\section{AB0158 DECREASED AUTOPHAGY IN SALIVARY GLANDS OF PRIMARY SJÖGREN'S SYNDROME PATIENTS COULD BE ASSOCIATED WITH AN INCREASED EXPRESSION OF INFLAMMATORY MARKERS}

María-José Barrera ${ }^{1}$, Sergio Aguilera ${ }^{2}$, Patricia Carvajal ${ }^{3}$, Isabel Castro ${ }^{4}$, Daniela Jara ${ }^{3}$, Sergio González ${ }^{5}$, Claudio Molina ${ }^{1}$, Soledad Matus ${ }^{6}$, MaríaJulieta González ${ }^{3}$. ${ }^{1}$ Facultad de Odontología, Universidad San Sebastián, Santiago, Chile; ${ }^{2}$ Clínica INDISA, Santiago, Chile; ${ }^{3}$ ICBM, Facultad de Medicina, Universidad de Chile, Santiago, Chile; ${ }^{4}$ Departamento Tecnología Médica, Universidad de Chile, Santiago, Chile; ${ }^{5}$ Escuela de Odontología, Universidad Mayor, Santiago, Chile; ${ }^{6}$ Fundación Ciencia and Vida, Santiago, Chile

Background: Salivary glands (SG) of primary Sjögren's syndrome (pSS) patients show high levels of IL-6 and endoplasmic reticulum (ER) stress ${ }^{1}$. In response to ER stress salivary epithelial cells trigger the unfolded protein response, seeking alleviate ER stress through several mechanisms, such as autophagy. The autophagy does not only eliminate misfolded protein aggregates, but also decreases the inflammation selectively removing proteins related to TLRs and inflammasomes. Interestingly, the activation of IL-6/JAK/STAT3 signaling pathway inhibits the autophagy through an increase in MCL-1 expression². Given the role of the autophagy as anti-inflammatory mechanism, it is interesting to evaluate if SG from pSS patients show a decrease in autophagy and if this correlates with the increased expression of inflammatory markers.

Objectives: To evaluate the expression of autophagy markers in labial SG (LSG) from pSS patients and 3D-acini deficient in autophagy. In addition, the expression of inflammatory marker IL-6, as well as, autophagy inhibitor MCL-1 was determined in 3D-acini control or deficient in autophagy, evaluating the possible participation of IL-6/JAK/STAT3 signaling pathway.

Methods: In LSG of 11 anti-Ro/La seropositive pSS patients and 10 control sub jects, mRNA levels of ATG5, mTOR and Beclin-1 were measured by qPCR ATG5, p62 and LC3B protein levels were measured by Western blot in LSG or 3D-acini deficient in autophagy by knocking down ATG5. HSG cells were transduced with lentiviral vectors expressing shRNAs against ATG5 mRNA or a control vector. Later 3D-acini were generated from shATG5 and control cells. Acini were incubated with $10 \mathrm{ng} / \mathrm{mL}$ recombinant IL- 6 in the presence or absence of $1.5 \mu \mathrm{M}$ of JAK inhibitor tofacitinib for $24 \mathrm{~h}$. The mRNA levels of IL- 6 and MCL- 1 were measured by qPCR.

Results: A significant decrease in mRNA levels of ATG5, mTOR and Beclin-1 was observed in LSG of SS-patients. In addition, a significant decrease of ATG5 protein levels together with an increase in $p 62$ receptor protein levels was determined in LSG from SS patients. In shATG5 3D-acini an increase in p62 protein levels was observed similar to LSG from SS patients. Also, an increase in mRNA levels of MCL- 1 was determined in 3D-acini stimulated with IL-6 and reverted with tofacitinib. Moreover, an increase in mRNA levels of IL- 6 was measured in shATG5 3D-acini compared to 3D-acini control.

Conclusion: Our results suggest an attenuation of autophagy in salivary epithelial cells from anti-Ro/La seropositive pSS patients, which could be associated with an increased expression of inflammatory markers. We postulate that a plausible via involved in the autophagy attenuation in LSG from pSS patients could be the IL-6/JAK/STAT3 pathway, which induces MCL-1 expression. Further experiments are needed to elucidate this proposal.

\section{REFERENCES}

[1] Barrera, MJ., et al. Autoimmun Rev, 2018. 17(8):p.796-808.

[2] Qin, B., et al. Sci Rep, 2015. 5: p. 15701.

Acknowledgement: Fondecyt-Postdoctorado-3170023, Fondecyt-1160015, Fondecyt-Iniciación-11170049.

Disclosure of Interests: None declared

DOI: 10.1136/annrheumdis-2019-eular.4740 